\title{
Effect Of Cupping Massage And Modified Spinal Decompression Therapy With Exercise Therapy In Neck Radiculopathy Syndrome
}

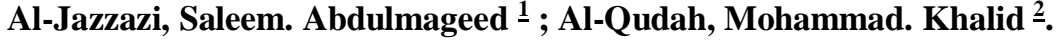 \\ 1 Associate Professor at the Faculty of Sport Science - Sport Training Department. Mutah University. \\ ${ }^{2}$ Associate Professor at the Faculty of Sports Science - Sport Rehabilitation Department. Mutah University. \\ Tel,: +962790201815; E-mail: yes22r@yahoo.co.uk PO Box 7 Mutah-Karak, Jordan.
}

Article History: Received: 20 February 2021; Revised: 10 March 2021; Accepted: 27 March 2021; Published online: 16 April 2021

\begin{abstract}
BACKGROUND: Cervical radiculopathy Syndrome (CRS) is a common neuro-musculo-skeletal disorder causing pain and disability. Manual therapy interventions including cervical traction with other treatment modalities have been advocated to decrease pain and disability caused by cervical radiculopathy (CR).Al-Qudah \& AL-Jazzazi (2021) conducted a new method of Spinal Decompression Therapy (SDT) in patients with Chronic Lumbar Disc Herniation (CLDH) which includes Combination of Lumbar Traction With Cervical Traction (CLTCT) as one intervention. Despite of that this new method clinically reduces pain and disability more effectively than the conventional types of Traction, CLTCT method was not previously used in CR patients nor with Cervical Disc Herniation (CDH). The clinical effectiveness of this new method with other treatment modalities in patients with CRS was not approved yet. OBJECTIVE: The purpose of the presented work is to identify the effectiveness of rehabilitative program on patients with Cervical Radiculopathy, by (15) sessions for (4) Weeks. The suggested Rehabilitative program consisted of:1.Supine Soft Full Back, Shoulders and Neck Cupping Massage (CM) for (10) minutes, 2.CLTCT: Combined Lumbar Traction with Cervical Traction as one intervention for (20) minutes,3.Gradual Therapeutic Exercise Package of Neck Stretching and Strengthening Exercise for approximately (15) minutes. METHODS: In this study, Five outdoor male patients had accepted to participate and were randomly chosen from Al-Karak Governmental Hospital, All subjects applied the proposed rehabilitative program. The results were analyzed using the SPSS system. RESULTS: indicates that there was statistically significant difference between the pre and post measurements in favor of the post measurements in terms of Pain, Disability. CONCLUSIONS: The present study demonstrated that the use of proposed rehabilitative program has a positive effect on patients with Cervical Radiculopathy.
\end{abstract}

Keywords: Cervical Radiculopathy Syndrome, Combined Lumbar Traction with Cervical Traction, Whole Spinal Decompression Therapy, Cupping Massage, Neck Stretching and Strengthening exercise, Pain Score, Neck Disability Index.

\section{Introduction}

Cervical radiculopathy Syndrome (CRS) is a costly common neuro-musculo-skeletal disorder causing pain and disability [11,21]. CRS is the result of cervical nerve root pathology [35], and may be of compressive or inflammatory etiology, due to cytokines released due to disc herniation and rupture of the fibrous annuity. It may present as brachialgia, sensory alteration, motor alteration, scapular pain, and headache [36]. The annual incidence is 83.2 per 100,000, with an increase in prevalence through the fifth decade of life [13]. A more recent study from the US military found an incidence of 1.79 per 1000 person-years [20].

Guidelines suggest surgery in severe CRS for the rapid relief of pain in short terms [10]. Nonetheless, in all other conditions, conservative treatment should be preferred. In fact, patients undergoing conservative treatment have reported good to excellent outcomes in up to $90 \%$ of cases [32]. Conservative treatment of CRS has included different interventions such as manual therapy (mobilization and manipulation), physical therapy and traction [20].

Traction is part of the evidence based manual physical therapy management due to its mechanical nature, type of traction and parameters related to its applicability and are still to be explored more through research [11], it is typically recommended for patients with cervical radiculopathy [16]. Traction can be administered with different modality: mechanical or manual and in an intermittent or continuous mode, with the aim to increase intervertebral space, inhibit nociceptive impulses, and reduce spinal nerve root compression [13], and deliver nutrients and oxygen $[18,24,25]$.

According to Asiri, et al. (2020) [7] the advancements in traction equipment are needed to ensure their effectiveness in clinical situations, but actually advancements are extremely more needed in modifying creative new methods of Spinal Decompression Therapy (SDT) [3]. Al-Qudah\& Al-Jazzazi (2021) [3] had conducted a new modified method of SDT in patients with Chronic Lumbar Disc Herniation (CLDH), which was suggested by Al-Qudah, M.K. According to the authors, This method was named by Whole Spinal Decompression Therapy (WSDT) which includes Combination of Lumbar Traction With Cervical Traction (CLTCT) as one intervention [3]. Despite of that this modified method clinically reduced pain and disability more effectively than the conventional types of Traction, CLTCT was not previously used in CR patients nor with Cervical Disc 
Herniation $(\mathrm{CDH})$. The clinical effectiveness of this modified method with other treatment modalities in patients with CR was not approved yet. CLTCT tracts the spinal column upward and downward in the same time, it has a dual mechanical effect [3,5]. So, It is necessary to identify how CLTCT with other treatment modalities affect pain and disability in patients with Cervical Radiculopathy.

Ancient Chinese cupping therapy is globally wide used, While dry cupping is used as conservative treatments to release pain, disability, and also to improve range of motion. Cupping massage (CM) is more widely used method among Athletes [2], it also may increase skin and superficial tissues temperature in neck and back area $[33,19]$. CM could be beneficial as some deep therapeutic massage techniques $[4,3,2,19]$, and may inhibit cytokines and reduce inflammations effectively [14,15]. Al-Qudah \& Al-Jazzazi (2021) [3] reported that in the light of global Corona Pandemic restrictions, CM remains more safe than traditional therapeutic massage, CM prevents direct physical contact with patients.

Therapeutic exercise (TE) is one of the most common conservative treatments, It can assist the activation of the deep fibers through low loaded isometric activity [3,26,28]. TE seemed to decrease pain intensity and improve functional ability when added to the other treatment modalities [3,18,28]. Stretching is a common intervention performed during rehabilitation. Stretching is prescribed to increase muscle length and Range Of Motion (ROM), or to align collagen fibers during healing muscle [29]. Neck Stretching Exercise is widely used in CRS treatment, as well as Strengthening Exercises for increasing muscle power [9,11,16,21,25,27, 31, 34,37].

Effectiveness of traditional Traction Therapy when combined with other treatment modalities has been approved in CRS treatment, but the clinical effectiveness of Cupping Massage and CLTCT (combined Lumbar traction with Cervical traction as one intervention) with Exercise therapy Complex of Stretching and Strengthening exercise remains uncertain. previously It has not been used in CRS treatment. Therefore, the aim of the current study was to assess the efficacy of treatment of the suggested therapeutic program of [Cupping Massage, CLTCT (Combined Lumbar Traction with Cervical Traction as one intervention) and Gradual Therapeutic Exercise Package] in CRS rehabilitation in term of pain and disability.

\section{Methodology}

\subsection{Participants}

This study aimed to assess the suggested rehabilitative program efficacy [cupping massage, CLTCT :Lumbar traction combined with Cervical traction as one intervention and Gradual Therapeutic Exercise Package]in Cervical Radiculopathy Syndrome (CRS). Five outdoor male patients (mean age:40.6 \pm 7.536 ) where chosen from Al-Karak Governmental Hospital from $17^{\text {th }}$ January to $14^{\text {th }}$ February 2021. Subjects presented with CRS with radiating symptoms for over three months confirmed by report, were included in the study. Subjects who had infection with COVID-19 or had osteoporosis, vertebral fracture, previous back or neck surgeries, history of prolonged use of corticosteroids, artificial disc or other spinal implants, malignancy, in addition to neck pain due to other conditions such as Myelopathy were excluded from the study. All the subjects signed a written informed consent form prior to their participation in the study. The study was approved by the ethical committee of Ministry of Health and Health Care, Amman, The Hashemite Kingdom Of Jordan.

\subsection{Experimental design}

Subjects who fulfilled the criteria were assigned to the study as one experimental group. They applied the suggested rehabilitative program by (15) sessions for (4) Weeks: 1.Supine Soft Full Back and Neck CM for (10) minutes, 2. CLTCT: [Positional Lumbar Sustained Traction as Hip Flexion, Combined with Cervical Mechanical Sustained Traction, the hip was manually extra pulled and rotated, all was conducted as one intervention] for (20) minutes, 3.Gradual Therapeutic Exercise Package for total time (15) minutes approximately: [Stretching exercise for (6) minutes, Strengthening Exercise for (6) minutes and Supine Neck Extension Resistance Exercise for (1-3) minute. Outcome measures were assessed before and after the treatment period to measure pain intensity and daily ability with the scales outlined below.

\subsection{Outcome measures}

\subsubsection{Numerical Rating Scale (NRS)}

To estimate the pain score through selecting out of 10 , the number that best describes the intensity of pain, where 0 indicates "no pain" and 10 indicates "the worst possible pain".

\subsubsection{Neck Disability Index (NDI)}


Neck functional disability scale assessed the influence of Neck Pain on daily activities. The questionnaire contains ten questions addressing different aspects of daily living tasks. Each question scored from 0 to 5, higher value describes greater disability. The total scores were expressed in percentage.

\subsection{Interventions}

\subsubsection{Cupping Massage (CM)}

Simi prone position was used with special pillows for supporting the torso. Sterilization, lubrication and soft full back, shoulders and neck CM were used for 10 minutes. A disposable silicon cups was used measuring $3 \mathrm{~cm}$ and $6 \mathrm{~cm}$ to fit the back, shoulders and neck area, as described in AL-Qudah \& Al-Jazzazi (2021) [3]. Each subject received $\mathrm{CM}$ for 10 minutes per session by 15 sessions for 4 weeks.

\subsubsection{Combined Lumbar Traction With Cervical Traction (CLTCT)}

Supine Mechanical Sustained Cervical Traction (MSCT) was conducted via medical mobile cervical traction devise (Ospine-NTD-500), then immediately Supine Positional Sustained Lumbar Traction (PSLT) (Hip Flexion) (HP) was conducted, then the hip was manually extra pulled and rotated, then time recording started, as described in AL-Qudah (2021) [5]. This procedure was conducted as a method of Spinal Decompression Therapy (SDT) that aimed to execute a nonsurgical spinal decompression. Neck decompression force was manually controlled and raised gradually in the terminal part of the treatment. Each subject received approximately20 minutes of CLTCT per session by 15 sessions for 4 weeks. Forceful physical activities that compress the neck were prevented.

\subsubsection{Gradual Therapeutic Exercise Package (GTEP)}

Researchers has built the GTEP which was presented to a number of academic specialists to take their opinions. It included three groups of Exercise:

2.4.3.1. Stretching Exercise(SchE)that aimed to relax and restore length in the target group of neck muscles. SchE was conducted passively from sitting position, with neutral neck position. The formed angle was increased until pain appears. Each subject in the study received SchE for 6 minutes, by 1 minute for each exercise:

a. Neck SchE: bending to the Right.

b. Neck SchE: bending to the Left.

c. Neck SchE: bending Forward.

d. Neck SchE: bending Backward.

e. Neck Axial SchE: rotation to the Right.

f. Neck Axial SchE: rotation to the Left.

2.4.3.2. Strengthening Exercise (SthE) that aimed to restore power in the target group of neck muscles. SthE was conducted as Slight Low Loaded Isometric Resistance Exercise (SLLIRE) for continues 10 seconds with 5 seconds recovery in each time (round), by 4 times (rounds).All exercises were performed from sitting position, with neutral neck position. Subjects were asked to perform $10 \%$ to $75 \%$ of their power. The total time extended for 6 minutes, by 1 minute for each exercise:

a. Neck Resistance Exercise: bending to the Right.

b. Neck Resistance Exercise: bending to the Left.

c. Neck Resistance Exercise: bending Forward.

d. Neck Resistance Exercise: bending Backward.

e. Neck Axial Exercise: rotation resistance to the Right.

f. Neck Axial Exercise: rotation resistance to the Left.

2.4.3.3. Neck Extension Resistance Exercise (NERE) that aimed to correct and protect the cervical curvature during the exercise, as well as to restore power and endurance in back neck group muscles. NERE was conducted with high intensity as Isometric Resistance Exercise (HIIRE) for continues 10 seconds with 5 seconds recovery in each time (round), by 4-12 times (rounds). This exercises was performed from crook laying position, with neutral neck position. Cylindrical pillow was set under the neck arch, then subjects performed Neck Extension Resistance Exercise by pressing the head downward on the examination bed. The total time extended 
for 1-3 minutes. All participants were asked to perform the NERE three times daily as a home program in addition to one set during each session under the therapist supervision.

\subsection{Progression of performed Intensity in the suggested rehabilitation program which includes 3 phases} is described below:

- Phase 1: The first week by 4 sessions, approximately with total time 43 minutes:

1. Cupping Massage (CM).

2. Combined Lumbar Traction with Cervical Traction (CLTCT): during implementation of Supine Mechanical Sustained Cervical Traction (MSCT), pressure was raised gradually from 16 to 20 PSI, with Maximum Hip Flexion (HP) as Supine Positional Sustained Lumbar Traction (PSLT), with hip manually extra pulled and rotated.

3. Stretching Exercise (SchE) was conducted. including Strengthening Exercise (SthE) where subjects were asked to perform $10 \%$ of their power. Also Neck Extension Resistance Exercise (NERE) was performed for 4 rounds.

- Phase 2: The second week by 4 sessions, approximately with total time 44 minutes:

1. Cupping Massage (CM).

2. (CLTCT): during implementation of MSCT, pressure was raised gradually from 16 to 21 PSI, with Maximum HP as PSLT, with hip manually extra pulled and rotated.

3. Stretching Exercise (SchE) was conducted, including Strengthening Exercise (SthE) where subjects were asked to perform $50 \%$ of their power. Also Neck Extension Resistance Exercise (NERE) was performed for 8 rounds.

- Phase 3: The third and fourth weeks by 4 and 3 sessions per week respectively, approximately with total time 45 minutes:

1. Cupping Massage (CM).

2. (CLTCT): during implementation of MSCT, pressure was raised gradually from 16 to 22 PSI, with Maximum HP as PSLT, with hip manually extra pulled and rotated.

3. Stretching Exercise (SchE) was conducted, including Strengthening Exercise (SthE) where subjects were asked to perform 75\% of their power. Also Neck Extension Resistance Exercise (NERE) was performed for 12 rounds.

\section{Results}

\subsection{Statistical analysis}

The data were analyzed using statistics software SPSS version 22, significant values were set at $\mathrm{p}<0.05$.

Wilcoxon Signed Ranks Test was used to know the significance between pre and post intervention.

Five subjects completed the intervention (mean age: $40.6 \pm 7.53658$ ) in the study. Demographic and baseline characteristics of the subjects mentioned in Table 1.

Table No. (1) Descriptive Statistics

\begin{tabular}{|c|c|c|c|c|c|c|c|c|c|}
\hline & $\mathbf{N}$ & Minimum & Maximum & Mean & $\begin{array}{c}\text { Std. } \\
\text { Deviation }\end{array}$ & \multicolumn{2}{|c|}{ Skewness } & \multicolumn{2}{|c|}{ Kurtosis } \\
\hline & Statistic & Statistic & Statistic & Statistic & Statistic & Statistic & Std. Error & Statistic & Std. Error \\
\hline Age & 5 & 30.00 & 50.00 & 40.6000 & 7.53658 & -.285 & .913 & .079 & 2.000 \\
\hline Height & 5 & 1.73 & 1.80 & 1.7740 & .02702 & -1.339 & .913 & 2.021 & 2.000 \\
\hline Body Mass & 5 & 77.00 & 90.00 & 83.6000 & 5.12835 & -.171 & .913 & -1.169 & 2.000 \\
\hline BMI & 5 & 25.54 & 28.41 & 26.5480 & 1.13458 & 1.374 & .913 & 2.114 & 2.000 \\
\hline Pain Score & 5 & 5.00 & 6.00 & 5.2000 & .44721 & 2.236 & .913 & 5.000 & 2.000 \\
\hline Neck Disability & 5 & 30.00 & 34.00 & 31.6000 & 1.67332 & .512 & .913 & -.612 & 2.000 \\
\hline $\begin{array}{r}\text { Valid N } \\
\text { (listwise) }\end{array}$ & 5 & & & & & & & & \\
\hline
\end{tabular}

\subsection{Numerical Rating Scale}

The scores showed a significant change before and after the intervention with difference in Mean Ranks, Based on negative ranks: $(\mathrm{z}=-2.041)$ where ( $\mathrm{p}=.041)$ (showing5.2 $\pm .447-1.4 \pm .54772)$. The intervention showed an absolute change (Table 2) (Table 3). 


\subsection{Neck Disability Index (NDI)}

The scores showed a significant change before and after the intervention with difference in Mean Ranks, Based on negative ranks: $(\mathrm{z}=-2.121)$ where $\mathrm{p}=.034)$ ( showing $31.6 \pm 1.673-13.2 \pm 1.788)$. The intervention showed an absolute change(Table 2) (Table 3).

Table No.(2)

\begin{tabular}{|c|c|c|c|c|}
\hline Variables & Measurement & Mean & Std. Deviation & Std. Error Mean \\
\hline Pain Score & Pre & 5.2000 & .44721 & .20000 \\
& & & & \\
& Post & 1.4000 & .54772 & .24495 \\
& & 31.6000 & 1.67332 & .74833 \\
Neck Disability & Pre & 13.2000 & 1.78885 & .80000 \\
\hline
\end{tabular}

Table No.(3) Wilcoxon Signed Ranks Test

\begin{tabular}{|c|c|c|c|c|c|c|}
\hline \multicolumn{5}{|c|}{ Ranks } & \multirow[b]{2}{*}{$\mathbf{Z}$} & \multirow[b]{2}{*}{ Asymp. Sig. (2-tailed) } \\
\hline & Variables & $\mathbf{N}$ & Mean Rank & Sum of Ranks & & \\
\hline \multirow{4}{*}{$\begin{array}{r}\text { Pre-post Pain } \\
\text { Score }\end{array}$} & Negative Ranks & $5^{\mathrm{a}}$ & 3.00 & 15.00 & $-2.041^{b}$ & .041 \\
\hline & Positive Ranks & $0^{\mathrm{b}}$ & .00 & .00 & & \\
\hline & Ties & $0^{\mathrm{c}}$ & & & & \\
\hline & Total & 5 & & & & \\
\hline \multirow{4}{*}{$\begin{array}{r}\text { Pre-post } \\
\text { Neck } \\
\text { Disability }\end{array}$} & Negative Ranks & $5^{\mathrm{d}}$ & 3.00 & 15.00 & $-2.121^{b}$ & .034 \\
\hline & Positive Ranks & $0^{\mathrm{e}}$ & .00 & .00 & & \\
\hline & Ties & $0^{\mathrm{f}}$ & & & & \\
\hline & Total & 5 & & & & \\
\hline
\end{tabular}

\section{a. Wilcoxon Signed Ranks Test \\ b. Based on negative ranks.}

\section{Discussion}

The aim of the study was to evaluate how the suggested treatment program affect pain and disability in patients with Cervical Radiculopathy Syndrome (CRS).

The results showed that a 4-week of Cupping Massage (CM), Combined Lumbar Traction with Cervical Traction (CLTCT) and Gradual Therapeutic Exercise Package (GTEP) significantly reduced pain and disability. It was found that all patients who suffer from radiating pain in shoulders and brachial area and even pain radiating down to the medial distal fingers have relieved their symptoms completely at the first session, suggesting that treatment and reducing the pressure can prevent or relieve secondary problems such as brachialgia, sensory alteration, motor alteration, scapular pain and headache in Neck Radiculopathy Syndrome (CRS).

CM could relax superficial muscles, increase back and neck temperature and increase pain threshold $[2,3,4,5,19,30]$. On the other hand, Blood Letting or Wet Cupping (WC) may play major role in inhibiting cytokines and reducing inflammations [14,15], suggesting that applying CM for prolonged duration may inhibit cytokines and reduce inflammations in CRS, where this suggestion still uncertain and in need of answer. However, CM could facilitate other next modalities to act effectively [3,5].

Cervical Traction (CT) reduces the intradiscal pressure and vertically increases the intervertebral space and restore disc height allowing nutrients and oxygen supply to the disc [24], this relief of compression can promote regeneration process [6]. CLTCT method may relieve pain secondary to CRS more effectively than traditional CT [5]. CLTCT has a dual mechanical effect, which tracts the vertebral column upward and downward at the same time [3,5], that may reduce the intradiscal pressure and vertically increase the intervertebral space and restore disc height more effectively than traditional CT which tracts the vertebral column upward only. However, this comparison still uncertain in CRS cases and in need for answer.

In addition, the effect of ET as GTEP that may provide stability and decrease the risk of subsequent disorder through the activation of deep muscles, which is useful in treating the pain, disability and limited Range Of Motion (ROM) in CRS [9,11,16,21,27,31,34,37]. Stretching is a common intervention performed during rehabilitation. Stretching is prescribed to increase muscle length and ROM, or to align collagen fibers during 
healing muscle [29]. Neck Stretching and Strengthening exercise are widely used in neck pain and CRS treatment, as well as Strengthening Exercises for increasing muscle power [9,11,16,21,31,34,37].

Results are in agreement with prior study of Cupping Massage (CM) in the treatment of Chronic Nonspecific Neck Pain[33]. where CM was associated with reduction of pain and disability. Also results are in agreement with prior study of Dry Cupping (DC) in treatment of Chronic Neck and Shoulder Pain[12], where DC was associated with reduction of pain and increase in skin surface temperature. On the other hand, results are also consistent with prior studies of CM in the treatment of nonspecific Low Back Pain (LBP) $[3,4,19]$, where CM reduce pain and disability. Saha, et al. (2017) [33] Included 50 patients with chronic neck pain, Significant improvement on the visual analogue scale and disability were found due to the efficacy of CM. Hong, et al. (2006) [19] studied the effect of CM on 70 patients with nonspecific LBP, Significant improvement on the visual analogue scale and daily ability were found. Suggests that pain relief can be related to stimulation of blood circulation which decrease muscle tone which may improve ROM, and increase pain threshold and increase superficial tissue temperature, that may stimulate Endorphin secretion, known by the natural pain killers mechanism.

The results are consistent with prior studies of Traction in the treatment of CRS [1,8,11,16,17,22,35,37] where reduction of pain intensity and disability were found. Bukhari, et al. (2016) [11] studied the effects of Mechanical versus Manual Traction in Manual Physical Therapy combined with segmental mobilization (SM) and exercise therapy (ET) in 36 Patients with CRS along 6 weeks by 3 days per week, results indicate that Mechanical Traction combined with SM and ET was more effectively as compared with manual traction combined with SM and ET in term of pain and disability. Fritz, et al. (2014) [16] also studied86 patients with CRS along 4 weeks, to identify the effectiveness of exercise, exercise with mechanical traction, and exercise with over-door traction, where Mechanical Traction combined with exercise was associated with significant improvement of pain after 6 Months, as well as significant improvement of pain and disability after 12 Months follow-up. Suggesting that Mechanical Traction when combined with Exercise is more effective in pain and disability reduction, It increase intervertebral space, inhibit nociceptive impulses effectively.

Afzal, et al. (2017) [1] studied 40 patients with CRS along 3 weeks, to identify the effectiveness of Manual Traction vas Manual Opening Technique, where similar significant results were found in Manual Traction with or without Manual Opening Technique in term of pain and disability. Young et al. (2009) [37] studied 81 patients with CRS along 5 weeks by 2 times per week, to identify the effectiveness of manual therapy and exercise, with or without the addition of cervical traction, where pain and disability reduction were found with or without the addition of cervical traction. Suggesting that Manual Traction and Manual Opening Technique have approximately similar therapeutic mechanical efficacy in CRS treatment. Therefore, guidelines suggest the addition of Exercise Therapy to Spinal Decompression Therapy methods such as Manual or Mechanical Traction, Manual Opening Techniques and other modalities in CRS treatment. In this study, ET was added to CLTCT to ensure more positive effects in term of pain and disability in patients with CRS.

On the other hand, Khan, et al. (2017) [22] studied 40 patients with CRS along 3 weeks, to identify the effectiveness of Supine versus Sitting position in Intermittent Traction in addition of Transcutaneous Electric Nerve Stimulation (TENS) and hot packs. Intermittent Traction in Supine position was associated with pain and disability reduction more than sitting position. Suggesting that neck muscles are to be more relax in Supine position than sitting position. Traction from Supine position facilitate the process of increasing intervertebral spaces more effectively. In the current study, Supine Position was applied in CLTCT.

Garg et al. (2019) [17] studied a case of CRS in 30-year old male patient with a sharp pain in the left shoulder and lateral side of the left arm and limited Cervical spine motion. Neck Gravity Traction on the bed was conducted for 1 minute6 times a day. After 3 weeks, the patient had completely relief from pain. Savva et al. (2012) [35] studied the effect of Cervical Traction with Neural Mobilization in a 52-yearold woman with CRS for 4 weeks, where pain, cervico-brachial pain and disability reduction was found. Suggesting that manual therapy including all types of traction and neural mobilization increase intervertebral space, inhibit nociceptive impulses, and reduce spinal nerve root compression.

Al-Qudah \& Al-Jazzazi (2021) [3] studied 9 patients with Lumbar Bulging Disc for 6 weeks, CM and CLTCT with Core Stabilization Exercise where associated with pain and disability reduction and improvement in Range Of Motion. Al-Qudah (2021) [5] Also studied the impact of CLTCT in Chronic Lumbar Disc Herniation, where CLTCT was associated with pain and disability reduction. Suggesting that CLTCT as a modified method of Spinal Decompression Therapy is effective in treatment low back pain as well as neck disorders such as CRS. CLTCT has a dual mechanical effect, which tracts the vertebral column upward and downward at the same time [3,5], that may reduce the intradiscal pressure and vertically increase the intervertebral space and restore disc height effectively.

Although Exercise Therapy (ET) might be beneficial for patients with CRS, ET alone might not be sufficient for best improvement in patients with serious physical limitations to functional recovery [11,31], It has been 
recommended that the combination of ET and other treatment protocols would be useful for patients with CRS $[9,11,16,21,27,31,34,37]$.

Bukhari, et al. (2016) [11], Fritz, et al. (2014) [16] and Young, et al. (2009) [37] had used ET in CRS treatment, Stretching and Strengthening Exercise were used as a part of treatment program. In this study, Stretching and Strengthening Exercise were used and Supine Neck Extension Resistance Exercise was extra used with seated Cylindrical pillow under the neck arch to correct and protect the cervical curvature, as well as to restore power and endurance in neck back group muscles during Exercising. Lee, et al. (2019) [23] recommended to support lumbar curvature during Traction, where they used Spinal Decompression Therapy via lumbar lordotic curvecontrolled traction (L-LCCT) in treatment patients with Lumbar intervertebral Disc Herniation. L-LCCT was associated with pain relief and disability reduction more than other types of Traction without Lumbar curvature support.

The previous studies have separately examined Cupping Massage (CM) with or without some methods of Spinal Decompression Therapy (SDT) as Cervical Traction (CT), CT with or without ET in patients with CRS or Chronic Neck Pain. While the current study examined the efficacy of combined MC and CLTCT as a new method of SDT, and ET as GTEP in one proposed Program. The findings of the current study show the positive effects of the suggested rehabilitative program in patients with CRS. There were few limitations of the present study, including that long-term effects of the treatment were not obtained due to the limitation of time under the light of global Corona Pandemic restrictions.

\section{Conclusions}

A combination of CM with modified SDT as CLTCT and ET as GTEP has proven to be significant to reduce pain and disability in subjects with CRS. The researchers recommend MRI for future studies, where it could be used as a post intervention assessment tool, which will warrant robust measurement of the vertical mechanical displacement along the longitudinal axes of the spinal column, and the mechanical displacement of the affected neural level in the cervical region. Also, the researchers recommend the use of CLTCT via any other available traction devices in most Spinal disorder.

\section{References}

1. Afzal, R; Ghous, M; Shakil Ur Rehman, S; Masood, T.Comparison between Manual Traction, Manual Opening technique and Combination in Patients with cervical radiculopathy: Randomized Control Trial. J Pak Med Assoc. (2019) Sep;69(9):1237-1241.

2. Al-Khararbah, S. (2018). Impact Of Dry Cupping On Non-specific Low Back Pain-Muscular Spasm Origin- Among Sport Science Collage Students. Unpublished Master Thesis, Mutah University, AlKarak, Jordan.

3. Al-Qudah, M.K. \& Al-Jazzazi, S.A. Impact Of Cupping Massage and Modified Spinal Decompression Therapy With Core Stabilization Exercise In Lumbar Bulging Disc Management. PSYCHOLOGY AND EDUCATION (2021) 58(3): 1978-1986 .http://psychologyandeducation.net/pae/index.php/pae/article/view/4163/3702

4. Al-Qudah, M.K. \& Al-Jazzazi, S.A. Impact Of Rehabilitative Program On Patients With Low Back Pain - Muscular Origin Among Sports Science Collage Students In Mutah University - Jordan. PSYCHOLOGY AND EDUCATION (2021) 58(2): 10056-10066. http://psychologyandeducation.net/pae/index.php/pae/article/vie /3942/3788.

5. Al-Qudah, M.K. Impact Of Combined Lumbar Traction With Cervical Traction In Chronic Lumbar Disc Herniation. Turkish Journal Of Computer and Mathematics Education.Vol.12 No. 6 (2021), 1124-1131.

6. Apfel CC, Cakmakkaya OS, Martin W, Richmond C, Macario A, George E, et al. Restoration of disk height through nonsurgical spinal decompression is associated with decreased discogenic low back pain: a retrospective cohort study. BMC Musculoskelet Disord. 2010; 11(1): 155.

7. Asiri, F; Tedla, JS; Alshahrani, MS. D; Ahmed, I; Reddy, RS; Gular, K. Effects of patient-specific three-dimensional lumbar traction on pain and functional disability in patients with lumbar intervertebral disc Prolapse. Nigerian Journal of Clinical Practice, Year 2020, Volume 23, Issue 4 [p. 498-502]. DOI: 10.4103/njcp.njcp_285_19 PMID: 32246656.

8. Ayub, A; Osama, M; Ahmad, S.Effects of active versus passive upper extremity neural mobilization combined with mechanical traction and joint mobilization in females with cervical radiculopathy: A randomized controlled trial.J Back Musculoskelet Rehabil. (2019).;32(5):725-730. doi: 10.3233/BMR170887.

9. Bertozzi L, Gardenghi I, Turoni F, Villafañe JH, Capra F, Guccione AA, Pillastrini P. Effect of therapeutic exercise on pain and disability in the management of chronic nonspecific neck pain: 
systematic review and meta-analysis of randomized trials. Phys Ther. 2013 Aug;93(8):1026-36. doi: 10.2522/ptj.20120412. Epub 2013 Apr 4.

10. Bono, C.M.; Ghiselli, G.; Gilbert, T.J.; Kreiner, D.S.; Reitman, C.; Summers, J.T.; Jamie, L.; Baisden, M.D.; John Easa, M.D.; Robert Fernand, M.D.; et al. An evidence-based clinical guideline for the diagnosis and treatment of cervical radiculopathy from degenerative disorders. SpineJ.2011, 11, 64-72.

11. Bukhari SR, Shakil-Ur-Rehman S, Ahmad S, Naeem A. Comparison between effectiveness of Mechanical and Manual Traction combined with mobilization and exercise therapy in Patients with Cervical Radiculopathy. Pak J Med Sci. 2016 Jan-Feb;32(1):31-4.

12. Chi, L-M; Lin, L-M; Chen, C-L; Wang, S-F; Lai, H-L; Peng, T-C.(2016).The Effectiveness of Cupping Therapy on Relieving Chronic Neck and Shoulder Pain: A Randomized Controlled Trial. Evidence-Based Complementary and Alternative Medicine. Volume 2016, Article ID 7358918, 7 pages. http://dx.doi.org/10.1155/2016/7358918https://www.hindawi.com/journals/ecam/2016/7358918/

13. Colombo, Claudio ; Salvioli, Stefano ; Gianola, Silvia; Castellini, Greta; Testa, Marco.Traction Therapy for Cervical Radicular Syndrome is Statistically Significant but not Clinically Relevant for Pain Relief. A Systematic Literature Review withMeta-Analysis and Trial Sequential Analysis. J. Clin. Med. 2020, 9(11), 3389; https://doi.org/10.3390/jcm9113389.

14. Ekrami, N; Ahmadian, M; Nourshahi, M; Shakouri,H. Wet-cupping induces anti-inflammatory action in response to vigorous exercise among martial arts athletes: A pilot study. Complementary Therapies in Medicine, Volume 56, January 2021, 102611.

15. Evgeni, R. \&Leonid, K. New is the well-forgotten old: The use of dry cupping in musculoskeletal medicine. Journal of Bodywork \& Movement Therapies (2016) 20, 173e17

16. Fritz JM, Thackeray A, Brennan GP, Childs JD. Exercise only, exercise with mechanical traction, or exercise with over-door traction for patients with cervical radiculopathy, with or without consideration of status on a previously described sub grouping rule: a randomized clinical trial.. J Orthop Sports Phys Ther. 2014 Feb;44(2):45-57. doi: 10.2519/jospt.2014.5065. Epub 2014 Jan 9.

17. Garg, P. Home Care Neck Traction for a Patient With Neck Pain and Cervical Radiculopathy Symptoms: A Case Report. J Chiropr Med. (2019) Jun; 18(2): 127-130.

18. Gaowgzeh, R, M; Chevidikunnan, M,F; BinMulayh, I A; Khan, F. Effect of spinal decompression therapy and core stabilization exercises in management of lumbar disc prolapse: A single blind and randomized controlled trial. Journal of Back and Musculoskeletal Rehabilitation; 33 (2020) 225-231. DOI 10.3233/BMR-171099 . IOS Press. https://pubmed.ncbi.nlm.nih.gov/31282394/

19. Hong, Y; Wu, J; Wang, B. The effect of moving cupping therapy on nonspecific low back pain. Chinese Journal of Rehabilitation Medicine (2006);21(3):340-343.

20. Iyer, S.; Kim, H.J. Cervical radiculopathy. Curr. Rev. Musculoskelet Med. 2016, 9, $272-280$.

21. Kay TM, Gross A, Goldsmith CH, Rutherford S, Voth S, Hoving JL, Brønfort G, Santaguida PL. Exercises for mechanical neck disorders. Cochrane Database Syst Rev. 2012 Aug 15;(8):CD004250. doi: 10.1002/14651858.CD004250.pub4.

22. Khan, RR; Awan, WA; Rashid, S; Masood, T. A randomized controlled trial of intermittent Cervical Traction in sitting Vs. Supine position for the management of Cervical Radiculopathy.Pak J Med Sci. 2017 Nov-Dec;33(6):1333-1338. doi:

10.12669/pjms.336.13851. https://pubmed.ncbi.nlm.nih.gov/29492054/.

23. Lee, CH; Heo, SJ; Park, SH; Jeong, HS; Kim, SY. Functional Changes in Patients and Morphological Changes in the Lumbar Intervertebral Disc after Applying Lordotic CurveControlled Traction: A Double-Blind Randomized Controlled Study.J. Medicina (Kaunas).2019 Dec 19;56(1):4. https://pubmed.ncbi.nlm.nih.gov/31861714/

24. Macario A, Pergolizzi JV. Systematic literature review of spinal decompression via motorized traction for chronic discogenic low back pain. Pain Pract. 2006; 6(3): 171-8.

25. Macario A, Richmond C, Auster M, Pergolizzi JV. Treatment of 94 outpatients with chronic discogenic low back pain with the DRX9000: a retrospective chart review. Pain Pract. 2008; 8(1): 11-7.

26. MacDonald DA, Moseley GL, Hodges PW. The lumbar Multifidus: does the evidence support clinical beliefs? Man Ther. 2006; 11(4): 254-63.

27. Moustafa I.M; .Diab A.A. Multimodal Treatment Program Comparing 2 Different Traction Approaches for Patients With Discogenic Cervical Radiculopathy: A Randomized Controlled Trial. Volume 13, Issue 3, September 2014, Pages 157-167

28. Owies, R; Mujalli, M. (2018). The Effect Of A Proposed Program Of Therapeutic Exercise In The Treatment Of Patients With Slipped Disc In The Lumbar Region. Jordanian Educational Journal, Volume (3), No (2), Article (3), P; 48-66.

29. Page, Phil. Current Concepts In Muscle Stretching For Exercise And Rehabilitation. Int J Sports Phys Ther. 2012 Feb; 7(1): 109-119. 
https://www.ncbi.nlm.nih.gov/pmc/articles/PMC3273886/

30. Pei-changXU;Shu-liCUI; Derrik, A.w; Chen,W; ShengXU; Lee, T. (2014). Preliminary observation on effect of cupping on the skin surface temperature of patients with back pain. World Journal of Acupuncture - Moxibustion Volume 24, Issue 4, 30 December 2014, Pages 59-61. https://www.sciencedirect.com/science/ article/abs/pii/S1003525715600307

31. Romeo A, Vanti C, Boldrini V, Ruggeri M, Guccione AA, Pillastrini P, Bertozzi L. Cervical Radiculopathy: Effectiveness of Adding Traction to Physical Therapy-A Systematic Review and MetaAnalysis of Randomized Controlled Trials. Phys Ther. 2018 Apr 1;98(4):231-242. doi: 10.1093/physth/pzy001.

32. Saal, J.S.; Saal, J.A.; Yurth, E.F. Nonoperative Management of Herniated Cervical Intervertebral Disc with Radiculopathy. Spine 1996, 21, 1877-1883.

33. Saha, F.J; Schumann, S; Cramer, H; Hohmann, C; Choi, K.-E; Rolke, R; Langhorst, J; Rampp, T; Dobos, G; Lauche, R. (2017). The Effects Of Cupping Massage In Patients With Chronic Neck PainA Randomized Controlled Trial. Complement Med Res2017;24:26-32. https://www.karger.com/Article/Fulltext/4548 72

34. Salt E, Wright C, Kelly S, Dean A.A systematic literature review on the effectiveness of non-invasive therapy for cervico-brachial pain. Man Ther. 2011 Feb;16(1):53-65. doi: 10.1016/j.math.2010.09.005. Epub 2010 Nov 12.

35. Savva C, Giakas G. The effect of cervical traction combined with neural mobilization on pain and disability in cervical radiculopathy. A case report.. Man Ther. 2013 Oct;18(5):443-6. doi: 10.1016/j.math.2012.06.012. Epub 2012 Jul 18.

36. Silva L.E.C.T; Almeida L.E.P.C.A. Update on Cervical Hernia Treatment: Conservative Management and Indications of Different Surgical Techniques. Rev Bras Ortop (Sao Paulo). 2021 Feb; 56 (1): $18-23$.

37. Young IA, Michener LA, Cleland JA, Aguilera AJ, Snyder AR. Manual therapy, exercise, and traction for patients with cervical radiculopathy: a randomized clinical trial. Phys Ther. 2009 Jul;89 (7): 632-42. doi: 10.2522/ptj.20080283. Epub 2009 May 21. 